RR 298

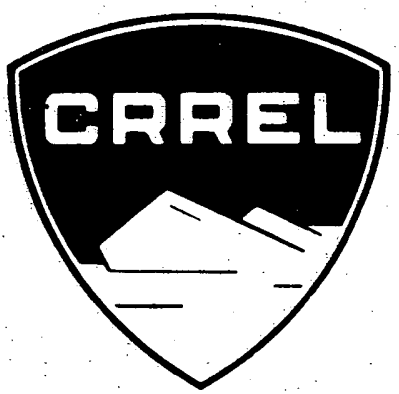

Research Report 298

\title{
MODEL ANALYSIS OF VEHICLE TRAFFICABILITY WITH APPLICATION TO SURFACE EFFECT VEHICLES ON SEA ICE FIELDS
}

Martin Smith and Yoshisuke Nakano

\author{
January 1972
}

\author{
PREPARED FOR \\ ADVANCED RESEARCH PROJECTS AGENCY \\ ARPA ORDER 1615 \\ B. \\ CORPS OF ENGINEERS, U.S. ARMY \\ COLD REGIONS RESEARCH AND ENGINEERING LABORATORY \\ HANOVER, NEW HAMPSHIRE
}




\section{PREFACE}

This report was prepared by First Lieutenant Martin L. Smith, Jr., Geologist, and Dr. Yoshisuke Nakano, Research Chemical Engineer, Physical Sciences Branch, Research Division, U.S. Army Cold Regions Ressearch and Engineering Laboratory. The report was prepared for the Advanced Research Projects Agency under ARPA Order 1615. It was technically reviewed by Dr. A. Assur, Mr. G. Frankenstein and Dr. W. Hibler, III, of USA CRREL.
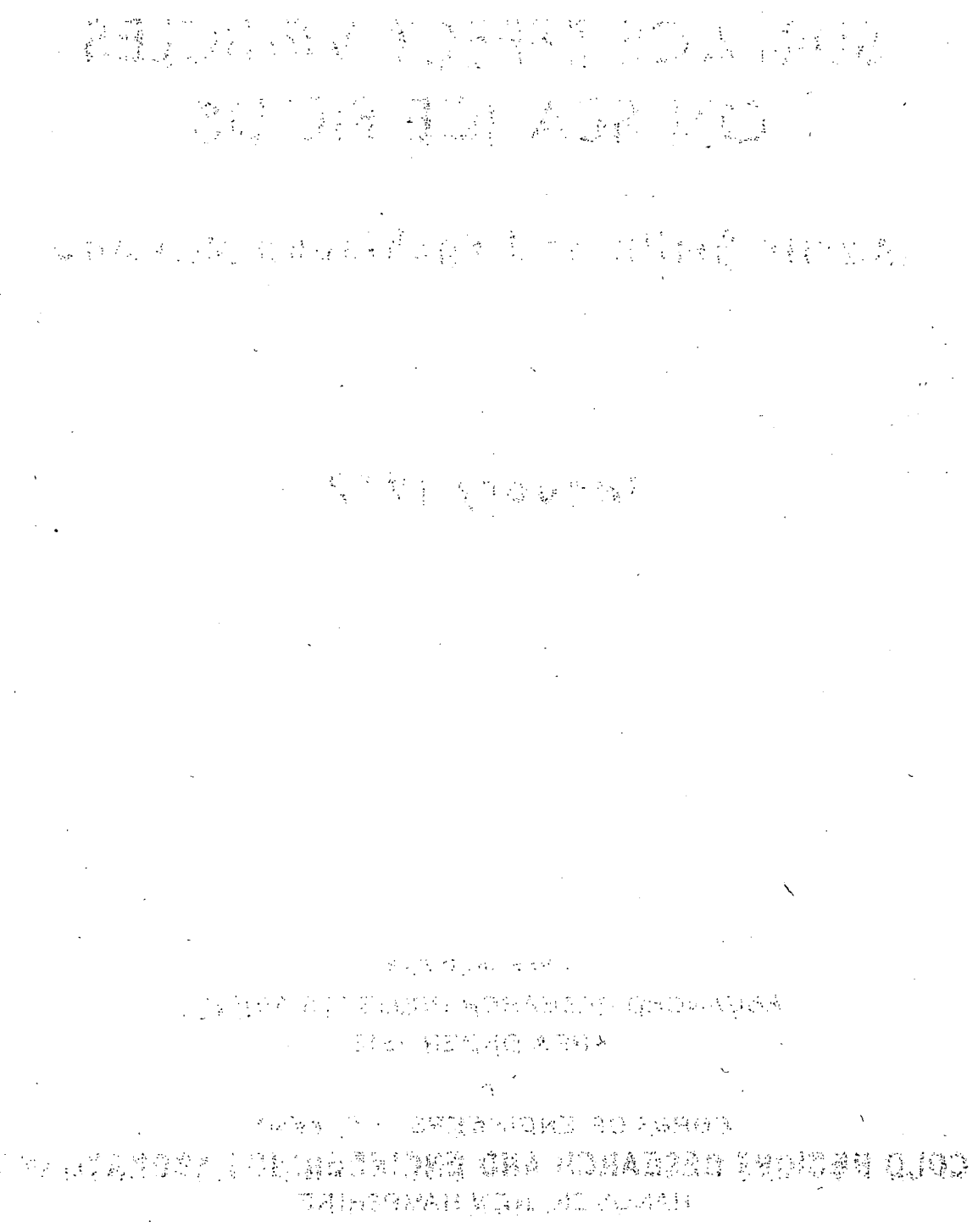


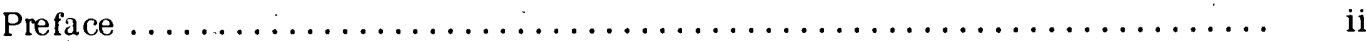

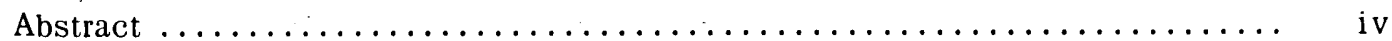

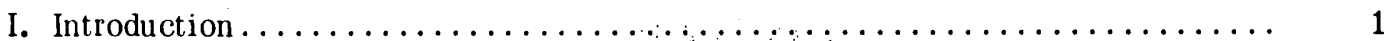

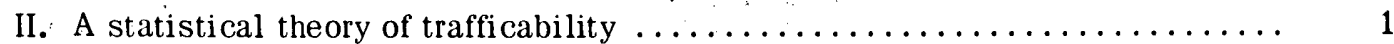

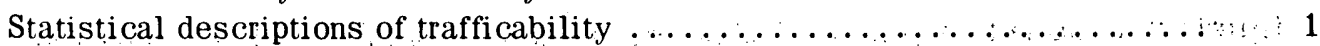

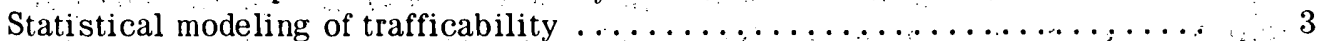

Trafficability in a variable space with comparison to a fixed space ......... 4

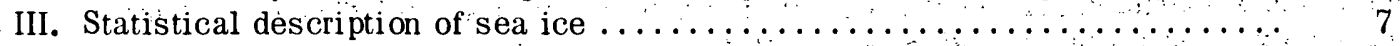

Homogeneous, isotropic, and normal random funetion $\ldots \ldots \ldots \ldots \ldots \ldots \ldots \ldots 7$

Upper bound of probability for finding an obstacle derived from two-point

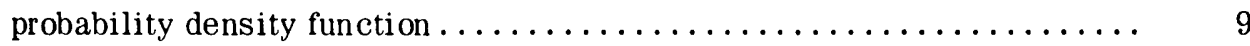

IV. Design criteria of a SEV derived from sea ice surface roughness . . . . . . 10

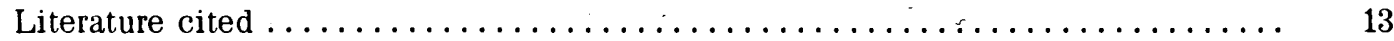

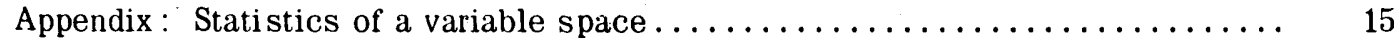

\section{ILLUSTRATIONS}

Figure

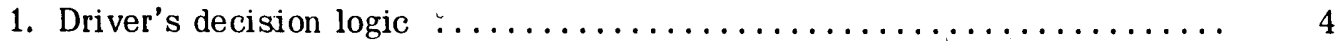

2. Typical vehicle paths in fixed obstacle space $\ldots \ldots \ldots \ldots \ldots \ldots \ldots \ldots . \ldots \ldots$

3. Survival rate vs trip length for various obstacle densities ........... 7

4. Correlation function for three typical samples $\ldots \ldots \ldots \ldots \ldots \ldots \ldots \ldots \ldots, 11$

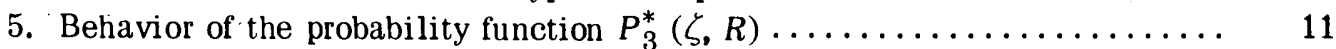

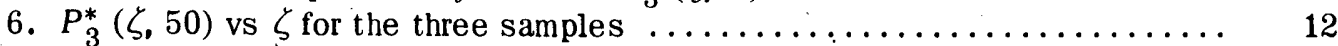

7. Expected relative extra travel time as a function of $P_{3}^{*}(\zeta, \mathrm{R}) \ldots \ldots \ldots \ldots, 12$

8. Probability of being trapped every 1000 miles vs $P_{3}^{*}(\zeta, 50) \ldots \ldots \ldots \ldots \ldots \quad 13$

\section{TABLES}

Table

I. Global statistics of a vehicle in a variable obstacle space $\ldots \ldots \ldots \ldots \ldots 6$

II. Penetration depth in a variable obstacle space $\ldots \ldots \ldots \ldots \ldots \ldots \ldots \ldots .6$

III. Survival rate vs $\mathrm{M} / \mathrm{M}^{*} \ldots \ldots \ldots \ldots \ldots \ldots \ldots \ldots \ldots \ldots \ldots \ldots \ldots \ldots \ldots \ldots$

IV. Comparison of time-of-arrival distribution's for variable and fixed spaces... 6 


\begin{abstract}
Efforts were made to derive the design criteria of surface effect vehicles operated on arctic sea ice. Statistical theories were developed to describe trafficability of the vehicles and topography of the sea ice. By the use of actual sea ice surface profiles obtained by an aerial laser profiler, the usefulness of the present statistical method was demonstrated.
\end{abstract}




\title{
MODEL ANALYSIS OF VEHICLE TRAFFICABILITY WITH APPLICATION TO SURF ACE EFFECT VEHICLES ON SEA ICE FIELDS
}

\author{
Martin Smith and Yoshisuke Nakano
}

\section{INTRODUCTION}

The utility of a surface vehicle is, in general, a function of the vehicle's capabilities, the terrain in which it is to be operated, the operator's skill, and the navigational aids available. Further, as a few minutes of driving in a snowstorm makes intuitively clear, even the most detailed knowledge of the above variables does not permit a sure (non-statistical) prediction of successful passage.

A straightforward, effective way of determining trafficability is repeated operation in repre-: sentative terrain. If, however, one is interested in developing a vehicle for a given terrain, experimental studies of this kind can be an expensive and time-consuming method of optimizing all the test vehicle's parameters.

A more fruitful technique, particularly in the early design stages, is to develop statistical models which enable us to relate various parameters of vehicle behavior to trafficability in a given suite of terrains. Such models may enable us to relate, for instance, SEV skirt height to trafficability in typical sea ice terrains. The extraction of statistical predictions of vehicle operation from both vehicle parameters and terrain data is the object of the present study.

Section II, together with the Appendix, develops a statistical theory of trafficability by reducing operator influence to a set of simple decision rules. The elimination of this imponderable allows us to develop a concise and straightforward theory and ensures that our predictions will be lower bounds for a vehicle's behavior.

Section III deals with the extraction of pertinent statistical parameters from profilometer data on the topography of sea ice. A technique based upon the theory of random functions is developed and applied.

Section IV combines the results of Sections II and III to predict a relation between SEV slopeclimbing capability and trafficability in sea ice terrains.

\section{A STATISTICAL THEORY OF TRAFFICABIITY}

\section{Statistical descriptions of trafficability}

A necessary prelude to mathematical models of trafficability is a quantitative notion of what trafficability is. What statistical parameters of a vehicle's performance are of interest to us in judging its utility in a given terrain? A number of candidates, such as mean velocity, time between breakdowns, etc., immediately present themselves. 
We shall consider, here, the time it takes a vehicle to travel a given distance in a given direction. From this definition of performance the following statistics naturally follow.

The mean time, of arrival is the time we would "expect" a vehicle to require to make the journey. It is a measure of the vehicle's operating efficiency in a given terrain. If there is a non-zero probability of a vehicle's not arriving at all we must be careful in our definition of this quantity.

The standard deviation of the time of arrival is another quantity of interest. It gives us a measure of the "spread" of arrival times we should anticipate and allows us to set "cutoff" times after which, for example, search and rescue operations should be initiated.

The lateral of fset is a measure of the offset of the vehicle from a direct path from origin to destination. Its size is a measure of the vehicle's inability to pursue a direct path between two points, save at the expense of increasing travel time.

The fatality rate is the probability that the vehicle will not successfully complete the assigned mission. It is a measure of the cost of a mission and aids the rational planning of rescue capabilities.

Suppose that we conduct a set of $N$ experiments in which vehicles are released along some given initial line and we measure the time $t_{\mathrm{i}}$ required for the $i^{\text {th }}$ vehicle to travel a distance $D$ away from the line. We suppose, further, that $K$ of the vehicles never arrive so that $t_{i}$ is defined only for $N-K$ values of $i$.

The mean time of arrival, then, is given by

$$
\bar{t}=\frac{1}{N-K} \sum_{i}^{*} t_{i}
$$

where $\sum_{i}^{*}$ denotes summation over the set of $N-K$ successful travelers.

The standard deviation of the time of arrival about the mean is given by

$$
\sigma=\left[\frac{1}{N-K-1} \sum_{i}^{*}\left(t_{i}-\bar{t}\right)^{2}\right]^{1 / 2}
$$

$N-K-1$ appears in the denominator instead of $N-K$ to correct for our use of $\bar{t}$ estimated from the same set of data.

Let $v$ be the mean velocity of the vehicles given by other considerations. Then

$$
t_{\min }=D / v
$$

is the minimum time in which a vehicle could make the trip, and

$$
\delta t=\bar{t}-t_{\min }
$$

is the "extra" time a vehicle spends traveling. If we assume that the additional time is spent in traveling in a random-walk fashion in a direction normal to the desired course, we may appeal to random-walk theory to estimate how far to the side of a straight-line path a vehicle is likely to emerge. The lateral off set $L_{0}$ is given by

$$
L_{0}=v \sqrt{\tau\left(\bar{t}-t_{\min }\right)}
$$


where $\tau$ is a characteristic length for the time interval between course alterations. $\tau$ may be thought of as the typical time between operator decisions regarding the vehicle's course.

The fatality rate is simply given by

$$
f=K / N
$$

and is the fraction of vehicles that are lost in transit.

\section{Statistical modeling of trafficability}

Each of these parameters is a function of the vehicle, the terrain, the operator, and the information available to the operator during transit. In order to model trafficability usefully, we must reduce this dependence to a small number of relevant parameters. We have chosen to do this in the manner described below.

We consider the vehicle's universe to be all points in the $x-y$ plane given by

$$
\vec{\xi}=m \hat{i}+n \hat{j}
$$

where $m$ and $n$ are integers and $\hat{i}$ and $\hat{j}$ are unit vectors in the $\mathbf{x}$ and $y$ directions. Each such point is, at any given time, either a "passable" point or an "obstacle" point. We may define a function, $\eta(\bar{\xi})$, over the plane such that

$$
\eta(\vec{\xi})= \begin{cases}0 & \text { if } \vec{\xi} \text { is passable } \\ 1 & \text { if } \vec{\xi} \text { is an obstacle }\end{cases}
$$

We also discretize time so thât $t=0,1,2, \ldots, \infty$.

At time $t=0$, the vehicle is introduced at some point along the line $y=0$. The vehicle is assigned the mission of proceeding "north" (the $\hat{j}$ direction) as efficiently as possible until it achieves the line $y=N$, at which time it ceases movement. During a unit time interval the vehicle may move one point east, north, or west of its previous location.

Let $\vec{\xi}_{t}$ denote a vehicle's position at time $t$. The nature of the vehicle's next move is determined by the values of $\eta$, the obstacle function, on the points east, north, and west of $\xi_{t}$. The precise move is determined by application of the following decision rules:

1) If $\vec{\xi}_{t}+\hat{j}$ is a passable point, the vehicle proceeds to it; otherwise it

2) determines if both of $\vec{\xi}_{t} \pm \hat{i}$ are passable and, so, chooses one randomly and moves to it; otherwise it

3) determines if one of $\vec{\xi}_{t} \pm \hat{i}$ is passable and, if so, moves to it; otherwise

4) the vehicle is trapped and is considered a fatality.

Under these rules, a vehicle is just barely able to avoid imminent collision, since information is available only about the immediately adjacent points. Furthermore, the vehicle cannot reverse. Figure 1 portrays the decision logic graphically.

We see that if $\eta(\vec{\xi})=0$ for all $\vec{\xi}$ the vehicle will proceed due north without deflection and will achieve its mission in the minimum possible time. As the density of obstacle points increases from zero, the vehicle's path becomes increasingly circuitous and its chance of entrapment greater.

We have, to date, considered two methods for assigning values to the obstacle function $\eta$. . The first of these we label the "scintillating" or "variable" model. At each time $t, \eta(\vec{\xi})$ for a given $\vec{\xi}$ is assigned the value one with probability $p$ and the value zero with probability $1-p$. In this model we have 

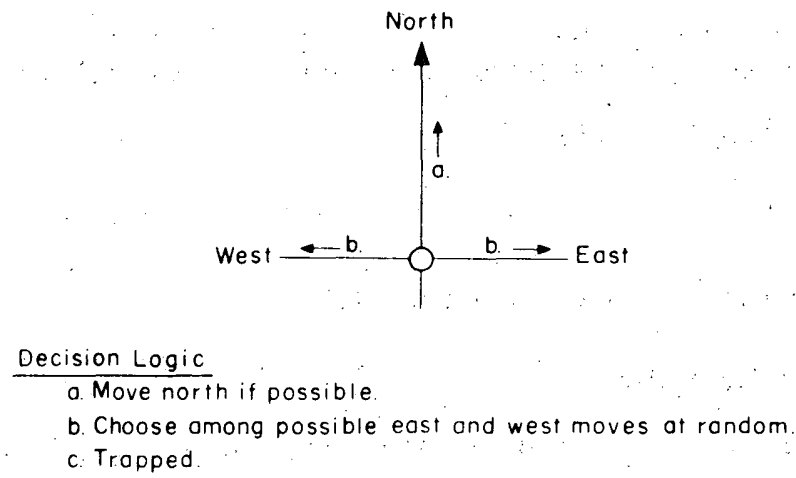

Figure 1. Driver's decision logic.

$$
E(\eta)=p
$$

where $E$ is the mathematical expectation operator over either space or time. In essence, the obstacle space is recreated at each time $t$, keeping only the density of obstacles constant. We have, to date, considered only spatially and temporally homogeneous distribution of obstacles.

The variable model simulates the effects of fog patches, drifting snow, etc. Furthermore, as discussed in the Appendix and summarized in the next section, we have been able to extract a number of useful analytic expressions from this model.

Because the behavior of the obstacle distribution is uncorrelated in time, the probability that a vehicle will be able to make any particular one of its three possible moves, or none of them, is independent of the vehicle's history. Consequently each move of a vehicle in a variable space is one sample of a Markov stochastic process.

The second method we have used for assigning $\eta$ differs from the above in that $\eta$ is determined only once, at time $t=0$, and remains fixed thereafter. We have labeled this the "fixed" model.

The fixed model is intended to simulate the effects of such obstructions as terrain features. Consequently, we consider it to be the more useful of the two.

The passage of a vehicle through any given fixed space is also a Markov process, but in a less useful sense. For this study we wish to find the statistics associated with the set of all. fixed grids of a given obstacle density. We have not attempted to develop any analytic results for this case and have resorted to numerical simulation.

Figure 2 portrays the passage of three vehicles through a particular fixed space. The examples shown are illustrative and consist of a vehicle which proceeds directly to its goal, a vehicle which becomes trapped, and a vehicle which requires three extra time steps to complete its mission. Note that the last vehicle's second move could have been into a cul-de-sac.

\section{Trafficability in a variable space with comparison to a fixed space}

We present here some analytic and numerical results. The detailed theory of trafficability in a variable space is contained in the Appendix.

Let $p$ denote the obstacle density and $K$ the number of north moves required for success. We take $\tau$ and $v$ to be unity for simplicity. (This scaling can always be achieved by an appropriate choice of units for time and length.). Then for a variable space we have

$$
\bar{t}=\frac{K}{1-p+p^{3}}
$$




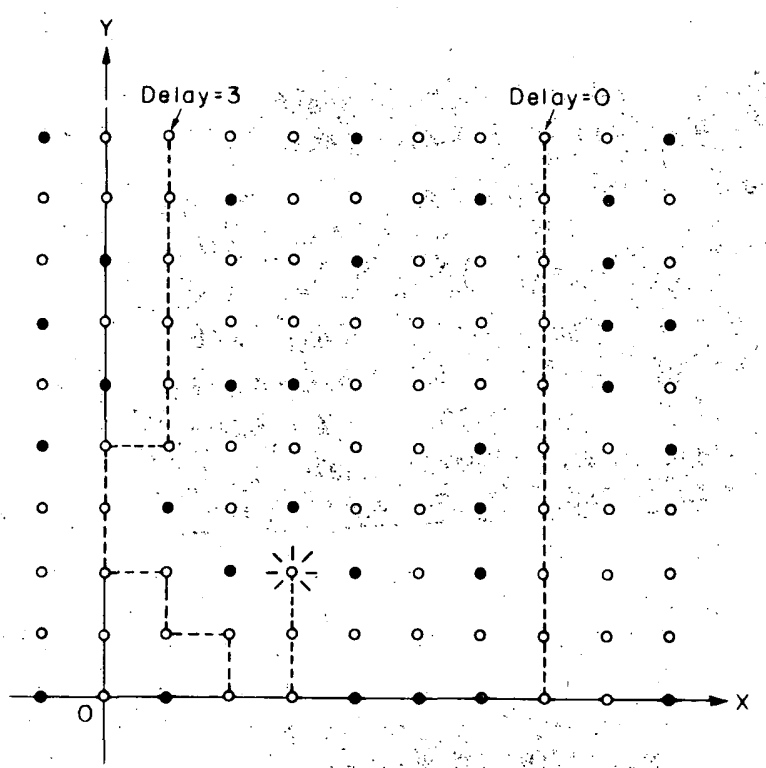

Figure 2. Typical vehicle paths in fixed obstacle space.

$$
\begin{aligned}
& \sigma=\sqrt{K\left(p-p^{3}\right)} /\left(1-p+p^{3}\right) \\
& L_{0}=2 \sqrt{K} \sqrt{\frac{p-p^{3}}{1-p+p^{3}}} \text { and } \\
& f=\left(\frac{1-p}{1-p+p^{3}}\right)
\end{aligned}
$$

We may also define the dimensionless incrementàl travel time,

$$
t^{+}=\frac{t-K}{K}
$$

which is the fractional increase in average arrival time. Clearly;

$$
E t^{+}=\bar{t}^{+}=\frac{p-p^{3}}{1-p+p^{3}}
$$

Since $\bar{t}^{+}$is independent of $K$, the mean relative extra travel time required does not increase with trip length. The deviation of $t^{+}$about its mean is given as

$$
E\left[\left(t^{+}-\bar{t}^{+}\right)^{2}\right]=\frac{1}{K}\left[\frac{P_{2}}{P_{0}}-\left(\frac{P_{1}}{P_{0}}\right)^{2}\right]
$$

where $P_{\mathrm{i}}$ are given by eq. $21-23$ for $i=0,1,2$ in the Appendix and are functions of $p$ only. The deviation of $t^{+}$decreases as $K$ increases, since each vehicle's path becomes more randomly uniform with increasing trip length.

Table I shows numerical results for these parameters for several values of $p$. The columns labeled $P_{0}, P_{1}$ and $P_{2}$ are explained in the Appendix. $K$ has been taken equal to 24. The columns labeled A.S. represent analytic values; those labeled M.C. were computed by Monte Carlo simulation. We have shown the survival rate $S_{M}$, defined as

$$
S_{\mathrm{M}}=1-t
$$

The rapid decrease of $S_{M}$ with obstacle density suggests that most results of practical interest will be associated with small values of $p(\leq 0.1)$ (or, alternatively, that our model is unrealistic). For $p=0.1$, we can see that the extra travel time $(\bar{t}-K)$ and the lateral of set are not large compared to the total travel time.

We define the penetration depth $M^{*}$ as the distance a vehicle has a $70 \%$ chance of ever going in a variable obstacle field of a given density. $M^{*}$ is given by.

$$
M^{*}=\log (0.7) / \log \left(\frac{1-p}{1-p+p^{3}}\right)
$$

Table II shows $\boldsymbol{M}^{*}$ for several values of $\boldsymbol{p}$. Note that $\boldsymbol{M}^{*}$ decreases very rapidly with increasing $\mathbf{p}$. Table III permits conversion to other survival rates. Thus, if we require a $99 \%$ chance of survival, a vehicle may proceed only 0.0282 penetration depths. In a $10 \%$ obstacle field, then, we are limited to a distance of 
Table I. Global statistics of a vehicle in a variable obstacle space.

\begin{tabular}{|c|c|c|c|c|c|c|c|c|c|c|}
\hline \multirow[b]{2}{*}{$p$} & \multicolumn{3}{|c|}{ Transition parameter } & \multicolumn{6}{|c|}{ Global parameter for $K=24$} & \multirow[t]{2}{*}{$L_{0}$} \\
\hline & $P_{0}$ & $P_{1}$ & $P_{2}$ & A.S.* & M.C.** & A.S. & M.C. & A.S. & M.C. & \\
\hline 0.1 & .9989 & 1.1086 & 1.3536 & .9737 & .9739 & 26.637 & 26.64 & 1.720 & 1.715 & 3.248 \\
\hline 0.2 & .9901 & 1.2254 & 1.8229 & .7876 & .7870 & 29.703 & 29.70 & 2.725 & 2.654 & 4.776 \\
\hline 0.3 & .9629 & 1.3244 & 2.3894 & .4032 & .4035 & 33.012 & 33.01 & 3.761 & 3.510 & 6.004 \\
\hline 0.4 & .9036 & 1.3609 & 2.9567 & .0878 & .0877 & 36. 144 & 36.10 & 4.909 & 4. 191 & 6.970 \\
\hline 0.5 & .8000 & 1.2800 & 3.3280 & .00472 & .00453 & 38.40 & 38.66 & 6.197 & 4.846 & 7.589 \\
\hline
\end{tabular}

Table II. Penetration depth in a variable obstacle space.

\begin{tabular}{lc}
$p$ & $M^{*}$ \\
\hline 0.0 & $\infty$ \\
0.1 & 356 \\
0.2 & 35.5 \\
0.3 & 9.46 \\
0.4 & 3.53 \\
0.5 & 1.60 \\
0.6 & 0.825 \\
0.7 & 0.467 \\
0.8 & 0.281 \\
0.9 & 0.169 \\
1.0 & 0.000 \\
\hline
\end{tabular}

Table III. Survival rate vs. $\mathbf{M} / \mathbf{M}$.

\begin{tabular}{cc}
$M / M^{*}$ & $S_{M}$ \\
\hline .00281 & .999 \\
.0282 & .99 \\
.0854 & .97 \\
.144 & .95 \\
.295 & .90 \\
.626 & .80 \\
1.00 & .70 \\
1.94 & .50 \\
3.38 & .30 \\
4.51 & .20 \\
6.45 & .10 \\
8.40 & .05 \\
9.83 & .03 \\
12.91 & .01 \\
19.37 & .001 \\
\hline
\end{tabular}

Table IV. Comparison of time-of-arrival distributions for variable and fixed spaces. $M=24$

\begin{tabular}{|c|c|c|c|c|c|c|c|c|c|}
\hline $\begin{array}{l}\text { Time of } \\
\text { arrival }\end{array}$ & Variable & $\begin{array}{l}p=0.02 \\
\text { Fixed* }\end{array}$ & R.E.\% & Variable & $\begin{array}{r}0.05 \\
\text { Fixed }\end{array}$ & R.E.\% & Variable & $\begin{array}{r}0.10 \\
\text { Fixed* }\end{array}$ & R.E.\% \\
\hline 24 & .6157 & .5892 & 4.5 & 2903 & .2764 & 5.0 & .0812 & .0785 & 3.4 \\
\hline 25 & 2960 & 3159 & 6.3 & 3459 & .3549 & 2.5 & .1890 & .1941 & 2.6 \\
\hline 26 & .0731 & .0754 & 3.0 & .2203 & .2133 & 3.3 & .2324 & .2281 & 1.9 \\
\hline 27 & .0131 & .0136 & 3.7 & .0955 & .0873 & .9 .4 & 2022 & .1766 & 14.5 \\
\hline 28 & 0017 & .0031 & 44. & .0329 & .0321 & 2.6 & .1342 & .1094 & 22.7 . \\
\hline 29 & & & & 0089 & .0126 & 29. & .0753 & .0626 & 20.3 \\
\hline 30 & & & & .0022 & .0065 & & .0353 & .0356 & 9 \\
\hline 31 & & & & & & & .0154 & 0218 & 29.5 \\
\hline 32 & & & & & & & .0058 & .0134 & 56.9 \\
\hline
\end{tabular}

* Monte Carlo, $2.5 \times 10^{5}$ trials in a 24 level space. 


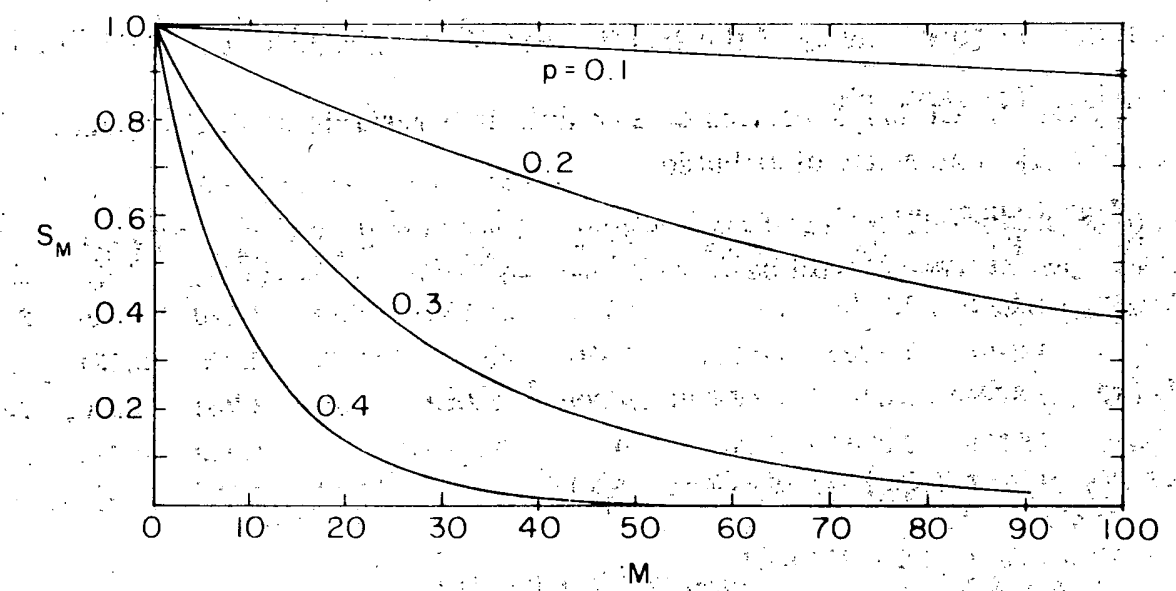

Figure 3. Survival rate vs. trip length

for various obstacle densities.

$0.0282 \times 356=10.04$

steps. Figure 3 shows' the rapid decrease in survival probability $S_{M}$ with increasing trip length.

Table IV shows the distribution of arrival times for both variable and fixed spaces of depth 24 , for $p=0.02,0.05$ and 0.10 . The column labeled "R.E.\%" is the percent relative difference of the two adjacent entries. The comparison is, we think, quite satisfactory for those time intervals in which significant arrival probabilities are found.

These results suggest that the two models give usefully identical results for low obstacle densities. Since we are, in practical applications, interested only in low densities, we can apply the above analytic results to either model:

\section{STATISTICAL DESCRIPTION OF SEA ICE}

Many natural phenomena have been successfully described as stochastic processes rather than deterministic ones. One of the earliest and most typical examples of such a situation is to be found in the theory of Brownian motion, where each coordinate of the Brownian particle is a random function of time.

The height of the sea ice surface can also be considered as a random function of location and time. Hibler and LeSchack (1970) studied sea ice surface profiles obtained by an aerial laser profiler approximately 200 miles north of Point Barrow in. April 1968. By assuming the height of the sea ice surface to be a homogeneous and isotropic random function of location, they obtained the correlation function and power spectral density.

We extend their work further to obtain a statistical description of the sea ice terrain, which can be directly connected to the trafficability of the SEV.

\section{Homogeneous, isotropic, and normal random function}

We introduce a random function $\mathbf{H}(\mathbf{x})$ defined by

$$
\mathbf{H}(\mathbf{x})=\text { height of sea ice surface }
$$

where $\mathbf{x}=$ a point $\left(\mathbf{x}^{(1)}, \mathbf{x}^{(2)}\right)$ in 2-dimensional Cartesian coordinates. 
In addition to requiring homogeneity of the function $\mathbf{H}(\mathbf{x})$, we introduce two assumptions; namely,

Assumption 1: $\mathrm{H}$ is isotropic

Assumption 2: $\mathbf{H}$ is normally distributed

These two assumptions have not been validated; however, if one considers a large area of sea ice terrain these assumptions should be a good approximation. We define the mean value and correlation functions as

$$
\begin{array}{ll}
E \mathbf{H}(\mathbf{x})=m(\mathbf{x}) & \text { mean value function } \\
E\left\{\left[\mathbf{H}\left(\mathbf{x}_{\mathrm{i}}\right)-m\left(\mathbf{x}_{\mathrm{i}}\right)\right]\left[\mathbf{H}\left(\mathbf{x}_{\mathrm{j}}\right)-m\left(\mathbf{x}_{\mathrm{j}}\right)\right]\right\} \\
\quad B\left(\mathbf{x}_{\mathrm{i}}, \mathbf{x}_{\mathrm{j}}\right)
\end{array}
$$

where $E$ denotes a mathematic expectation operator.

Since $\mathbf{H}(\mathbf{x})$ is homogeneous and isotropic, $B$ is a function only of the distance between two points. We have, then,

$$
B\left(\mathbf{x}_{\mathrm{i}}, \mathbf{x}_{\mathrm{j}}\right)=\boldsymbol{B}(\boldsymbol{r})
$$

where

$$
r=\left|\mathbf{x}_{\mathbf{i}}-\mathbf{x}_{\mathbf{j}}\right|
$$

For a normal random function $\mathbf{H}(\mathbf{x})$, the mean value $m$ and the correlation function $B$ completely specify $\mathbf{H}(\mathbf{x})$, i e. they determine all the multivariate probability functions (Yaglom, 1962).. The multivariate probability density functions are constructed in the following way (Prabhu, 1965). If the covariance matrix $\Gamma$ with element $\gamma_{i j}=B\left(\mathbf{x}_{\mathrm{i}}, \mathbf{x}_{\mathrm{j}}\right)$ is known and is nonsingular, then the multivariate probability density function $t_{\mathrm{n}}\left(h_{1}, h_{2}, \ldots, h_{\mathrm{n}} ; \mathrm{x}_{1}, \mathrm{x}_{2}, \ldots, \mathrm{x}_{\mathrm{n}}\right)$ is given as

$$
t_{\mathrm{n}}=\frac{|\Lambda|^{1 / 2}}{(2 \pi)^{n / 2}} \exp \left\{-\frac{1}{2} \sum_{i, j}^{n} \sum_{1} \lambda_{i j}\left(h_{i}-m_{i}\right)\left(h_{j}-m_{j}\right)\right\}
$$

where

$$
\begin{aligned}
& \Lambda=\Gamma^{-1}=\left(\lambda_{\mathrm{ij}}\right), \\
& |\Lambda|=\text { determinant of } \Lambda, \\
& h_{\mathrm{i}}=\mathbf{H}\left(\mathbf{x}_{\mathrm{i}}\right), \quad \text { and } \\
& \mathrm{m}_{\mathrm{i}}=\boldsymbol{E} \mathbf{H}\left(\mathbf{x}_{\mathrm{i}}\right)
\end{aligned}
$$

As a special case, the two-point probability density function $f_{2}\left(h_{1}, h_{2} ; x_{1}, x_{2}\right)$ is given as

$$
t_{2}=\frac{|\Lambda|^{1 / 2}}{2 \pi} \exp \left\{-\frac{1}{2} \quad \sum_{\mathrm{i}, \mathrm{j}=1}^{2} \sum_{\mathrm{ij}}\left(h_{\mathrm{i}}-m_{\mathrm{i}}\right)\left(h_{\mathrm{j}}-m_{\mathrm{j}}\right)\right\}
$$


where

$$
\begin{aligned}
& \lambda_{11}=\gamma_{22} / \mathrm{d} \\
& \lambda_{12}=\lambda_{21}=-\gamma_{12} / d \\
& \lambda_{22}=\gamma_{11} / d \\
& d=\gamma_{11} \gamma_{22}-\gamma_{12}^{2}
\end{aligned}
$$

Since $\mathbf{H}(\mathbf{x})$ is homogeneous and isotropic, we obtain

$$
\begin{aligned}
& t_{2}\left(h_{1}, h_{2} ; r\right)=\frac{1}{2 \pi B(0) \sqrt{1-\beta^{2}}} \exp \left[-\frac{1}{2 B(0)\left(1-\beta^{2}\right)}\right. \\
& \left.\left\{\left(h_{1}-m\right)^{2}+\left(h_{2}-m\right)^{2}-2 \beta\left(h_{1}-m\right)\left(h_{2}-m\right)\right\}\right]
\end{aligned}
$$

where

$$
\beta=\ddot{B}(\mathrm{r}) / B(0) \cdot
$$

\section{Upper bound of probability for finding an obstacle derived from two-point probability density function}

Although a complete quantitative description of the terrain capabilities of an SEV has not yet been obtained, it is known that the slope of the surface and sudden changes in sea ice height are two of the most important controlling factors. We characterize this situation as follows. Suppose we are operating the vehicle at a certain location, and we are interested in the probability of finding an obstacle within a certain distance $r$ of the present location, $x=x_{1}$. We begin by computing the probability that $\mathbf{H}\left(\mathrm{x}_{1}\right)=h_{1}$ and $\mathbf{H}\left(\mathrm{x}_{2}=\mathrm{x}_{1}+r\right) \geq h_{1}+\zeta$; namely,

$$
\begin{aligned}
& P_{1}^{*}\left\{\mathrm{H}\left(\mathrm{x}_{1}\right)=h_{1}, \text { and } \mathrm{H}\left(\mathrm{x}_{2}\right) \geq h_{1}+\zeta\right\} \\
& \int_{h_{1}+\zeta}^{\infty} l_{2}\left(h_{1}, h_{2} ; r\right) d h_{2} \\
& =C \exp \left[-D\left(1-\beta^{2}\right)\left(h_{1}-m\right)^{2}\right] \sqrt{\frac{\pi}{D}}[1-\phi(s)]
\end{aligned}
$$

where

$$
\begin{aligned}
C & =1 /\left[2 \pi B(0) \sqrt{\left.1-\beta^{2}\right]}\right. \\
D & =1 /\left[2 B(0)\left(1-\beta^{2}\right)\right] \\
s & =\sqrt{2 D}\left\{\left(h_{1}-m\right)(1-\beta)+\zeta\right\} \\
\phi(s) & =\frac{11}{\sqrt{2 \pi}} \int_{-\infty}^{x} \exp \left(-\frac{t^{2}}{2}\right) d t
\end{aligned}
$$


For SEV operation the difference in the heights of two neighboring locations is important rather than the absolute height of the surface, so we compute the following probability:

$$
\begin{aligned}
P_{2}^{*} \quad\left\{\mathbf{H}\left(\mathrm{x}_{2}\right) \geq \mathbf{H}\left(\mathrm{x}_{1}\right)+\zeta ; r=\left|\mathrm{x}_{1}-\mathrm{x}_{2}\right|\right\} \\
=P_{2}^{*}(r, \zeta) \\
=1-\frac{1}{\sqrt{2 \pi B(0)}} \int_{-\infty}^{\infty} \exp \left\{-\frac{1}{2 B(0)}\left(h_{1}-m\right)^{2}\right\} \\
\quad \phi\left[\frac{1}{\sqrt{B(0)\left(1-\bar{\beta}^{2}\right.}}\left\{(1-\beta)\left(h_{1}-m\right)+\zeta\right\}\right] d h_{1}
\end{aligned}
$$

The function $P_{2}^{*}(r, \zeta)$ is the probability that height of the two points separated by a distance $r$ differs more than $\zeta$.

Finally we compute the probahility $P_{3}^{*}$ that the condition $\mathbf{H}\left(\mathrm{x}_{1}\right) \geq \mathbf{H}\left(\mathrm{x}_{1}\right)+\zeta$ is ever satisfied within a specified distance $R . P_{3}^{*}$ is given as

$$
P_{3}^{*}(\zeta, R)=\frac{1}{R} \int_{0}^{\mathrm{R}} P_{2}^{*}(r, \zeta) \mathrm{d} r
$$

If we assume that the SEV is designed to pass over an ice ridge of up to $\zeta$ (ft), the function $P_{3}^{*}(\zeta, R)$ gives an upper bound on the probability of finding an obstacle within the distance $R$. $P_{3}^{*}\left(\zeta_{;} R\right)$ can also be interpreted as an upper bound on the fraction of points in an obstacle state $p$ as described in Section II, if the distance is properly chosen.

\section{DESIGN CRITERIA OF A SEV DERIVED FROM SEA ICE SURFACE ROUGHNESS}

We used the correlation functions obtained by Hibler and LeSchack (1970) from natural sea ice. Figure 4 shows three samples of correlation functions. We computed the values of $P_{3}^{*}(\zeta, R)$ for Sample 1 to examine the general behavior of $P_{3}^{*}$. The results of this computation are shown in Figure 5, in which the values of $P_{3}^{*}$ are plotted as a function of $R$ with $\zeta$ being a parameter. $P_{3}^{*}$ naturally increases with distance and decreases rapidly as $\zeta$ increases.

As we mentioned in Section III, if we choose the distance $R$ properly, $P_{3}^{*}$ can be interpreted as an upper bound of the fraction of points in an obstacle state $p$, as defined in Section II. We chose the distance $R$ to be $50 \mathrm{ft}$ as a trial value and computed $P_{3}^{*}(\zeta, 50)$ for all three samples. The results of this computation are shown in Figure 6 , in which $P_{3}^{*}$ is plotted against $\zeta$. It is easy to see from this figure that Sample 3 is the most difficult terrain of the three. We note that even for Sample 3 , if $\zeta \geq 2.0(\mathrm{ft}), P_{3}^{*}(\zeta, 50)$ is less than $10^{-2}$ or $1 \%$.

In order to connect the results of Section II with the present analysis, we plotted expected relative extra travel time $\bar{t}^{+}$versus $P_{3}^{*}(\zeta, 50)$ in Figure 7 . For instance if the total distance is 1000 miles and $P_{3}^{*}=0.1$, one has to travel 110 miles extra. However, if the SEV can pass over an ice ridge up to $2.5 \mathrm{ft}$ in height, the extra distance traveled would only be on the order of 10 miles. 


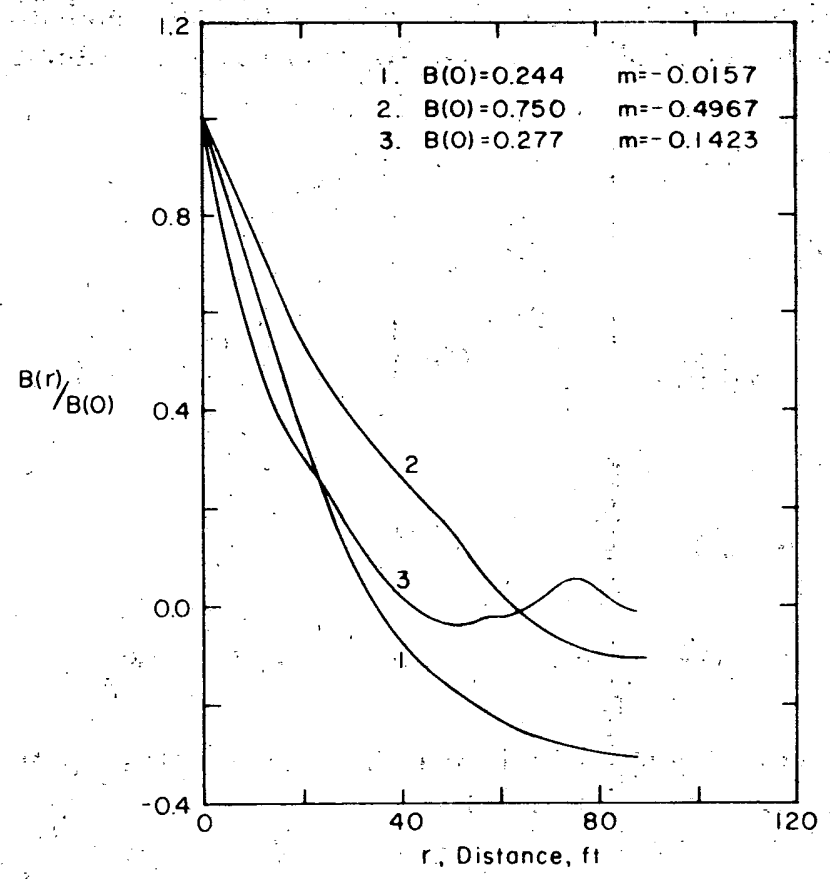

Figure 4. Correlation function for three typical samples.

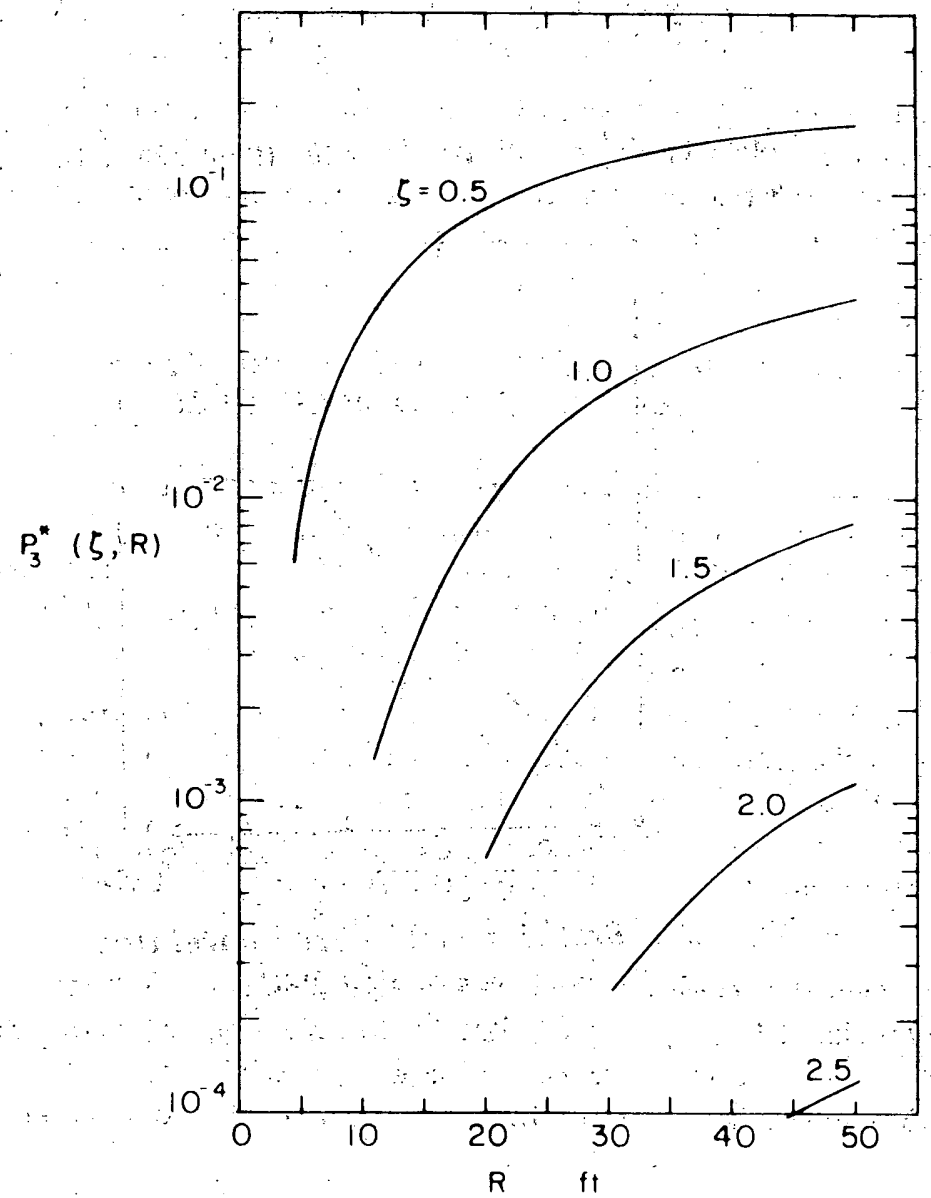

Figure 5. Behavior of the probability function $P_{3}^{*}(\zeta, R)$. 


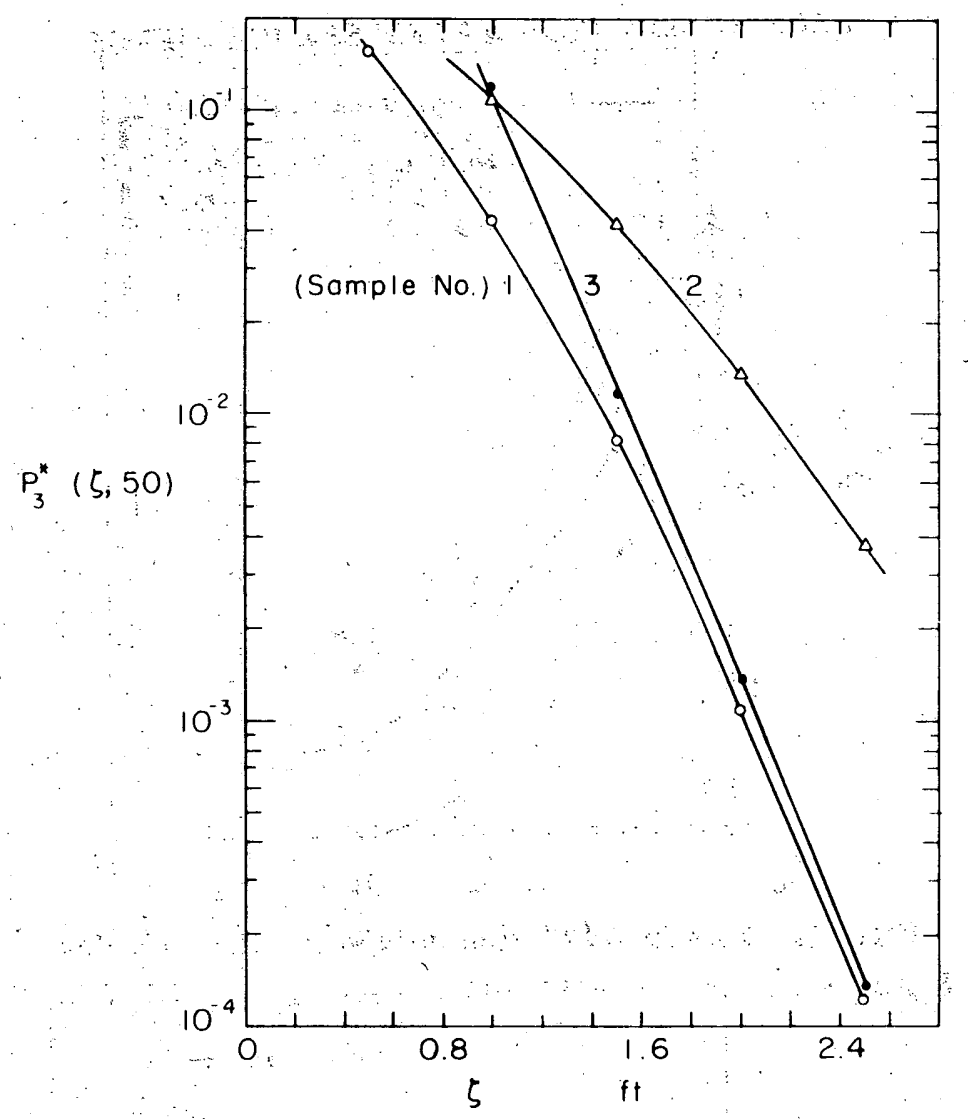

Figure 6. $P_{3}^{*}(\zeta, 50)$ vs. $\zeta$ for the three samples.

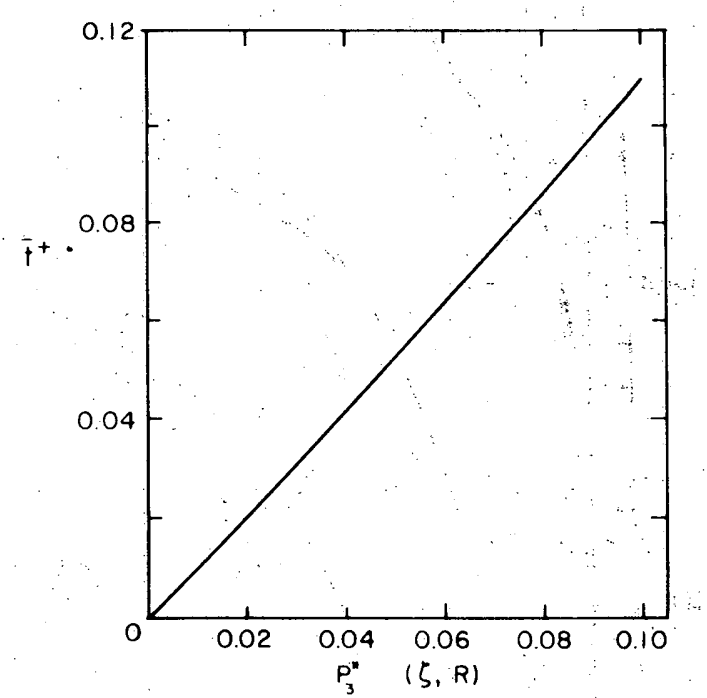

Figure 7. Expected relative extra travel time as a function of $P_{3}^{*}(\zeta, R)$. 


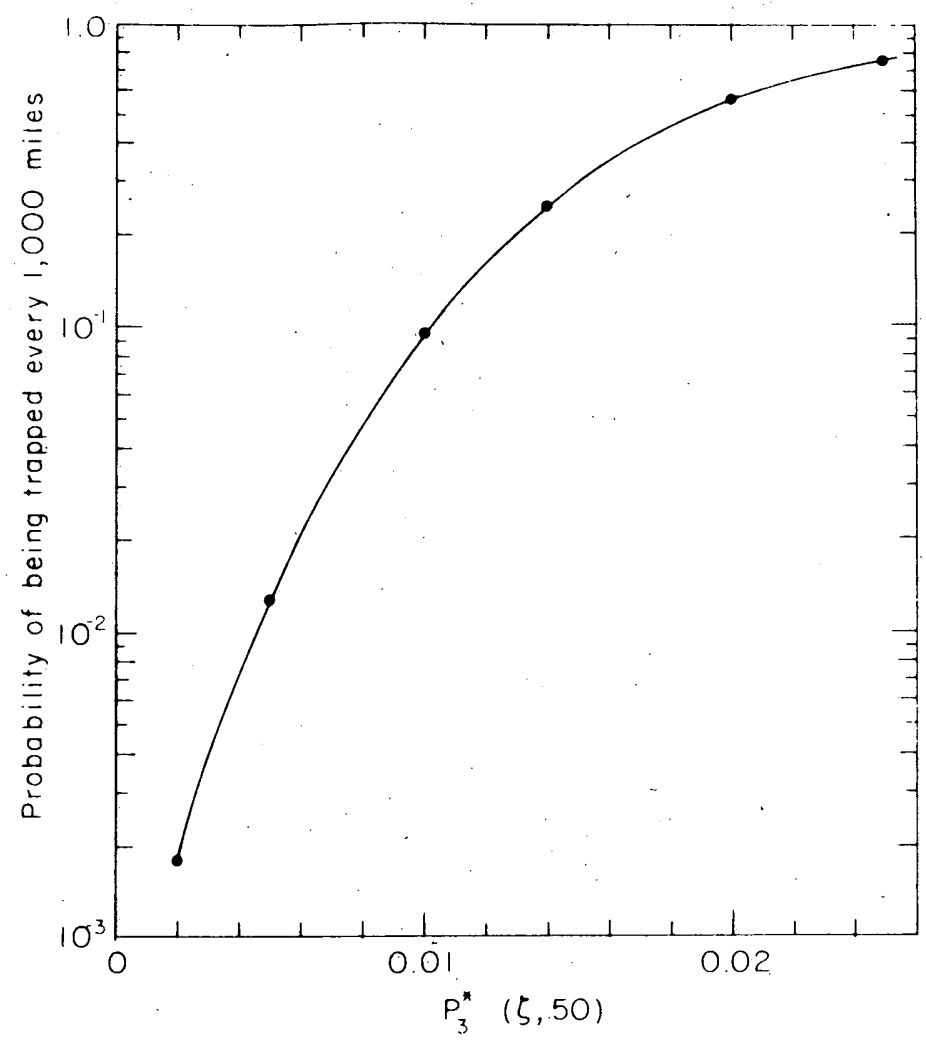

Figure 8. Probability of being trapped every 1000 miles vs. $P_{3}^{*}(\zeta, 50)$.

A severe restriction is imposed by the fatality rate. In Figure 8 we plotted the values of $\left(1-S_{M}\right)$, which is probability of being trapped, for every 1000 miles versus $P_{3}^{*}(\zeta, 50)$, where $S_{M}$ is computed as follows

$$
\begin{aligned}
S_{M} & =\left\{P_{3}^{*}(\zeta, 50)\right\}^{\frac{10^{3} \text { miles }}{50 \mathrm{It}}} \\
& =P_{3}^{* 10^{5}}
\end{aligned}
$$

If $P_{3}^{*}=0.01$, one out of every $100 \mathrm{SEV}$ 's traveling 1000 miles will be trapped in the field. In order to make $P_{3}^{*}<0.01$ the SEV should be able to pass over about 2.5 - $\mathrm{ft}$ ice ridges.

Alt hough our present model needs further refinements and only limited information on natural sea ice is available, the results of the analysis indicate that the SEV is a promising operational. vehicle in sea ice terrain, in view of the fact that a small prototype SEV is able to pass over ridges up to $3.0 \mathrm{ft}$ in height without difficulty. Since field studies on trafficability are often expensive and time-consuming, this model analysis may provide useful guidance to designers.

\section{LITERATURE CITED}

Hibler, W.D., III, and L.A. LeSchack (1970) Power spectrum analys is of undersea and surface sea ice ridge profiles. U.S. Army Cold Regions Research and Engineering Laboratory (USA CRREL) Technical Note.

Prabhu, N.U. (1965) Stochastic processes; basic theory and its applications. New York: Macmillan Company.

Yaglom, A.M. (1962) An introduction to the theory of stationary random functions. New Jersey: Prentice-Hall. 


\section{APPENDIX: STATISTICS OF A VARIABLE SPACE}

Let $P(N)$ denote the probability that a vehicle will make the northward transition from the line $y=j$ to the line $y=j+1$ after exactly $N$ moves. We assume $P(N)$ is independent of $j$, time, and the vehicle's east-west position. Clearly,

$$
P(N)=0 \cdot \quad \text { for } N \leqq 0
$$

We define the moments of $P(N), P_{0}, P_{1}, P_{2}, \ldots$ by

$$
P_{\mathrm{L}}=\sum_{\mathrm{N}=1}^{\infty} P(N) \quad N^{\mathrm{L}}
$$

Note that $P_{0}$ is simply the probability that the vehicle will ever make the transition.

Let $Q^{(K)}(N)$ denote the probability that a vehicle originating at $y=0$ at time zero will first achieve the line $y=K$ at exactly time $N$. Clearly

$$
Q^{(K)}(N)=0 \text { for } N<K
$$

We extend (3) to hold for negative $N$.

By considering all the ways to go from level $K-1$ to level $K$, we see that $Q^{(K)}(N)$ is related to $Q^{(K \cdot 1)}(N)$ by

$$
Q^{(K)}(N)=\sum_{M=1}^{\infty} Q^{(K-1)}(N-M) P(M)
$$

$P(M)$ thus plays the role of a transfer function.

We define the moments of $Q^{(K)}(N)$ as

$$
D_{\mathrm{L}}^{(K)}=\sum_{\mathrm{N}=1}^{\infty} Q^{(K)}(N) N^{\mathrm{L}}
$$

If we use (4) in (5), we have

$$
\begin{aligned}
D_{\mathrm{L}}^{(K)} & =\sum_{\mathrm{N}=1}^{\infty} \sum_{M=1}^{\infty} N^{L} Q^{(K-1)}(N-M) P(M) \\
& =\sum_{\mathrm{N}=1}^{\infty} \sum_{M=1}^{\infty}(N-M+M)^{L} Q^{(K-1)}(N-M) P(M) \\
& =\sum_{N=1}^{\infty} \sum_{M=1}^{\infty} \sum_{j=0}^{L}\left(\begin{array}{c}
L \\
j
\end{array}\right)(N-M)^{L-j} Q^{(K-1)}(N-M) M^{j} P(M)
\end{aligned}
$$


where $\left(\begin{array}{l}L \\ j\end{array}\right)$ is the binomial coefficient. We may reorder the sums to arrive at

$$
D_{\mathrm{L}}^{(K)}=\sum_{\mathrm{j}=0}^{\infty}\left(\begin{array}{l}
L \\
j
\end{array}\right) H_{\mathrm{L} j}
$$

where

$$
H_{\mathrm{Lj}}=\sum_{M=1}^{\infty} P(M) M^{\mathrm{j}} \sum_{\mathrm{N}=1}^{\infty} Q^{(K-1)}(N-M)(N-M)^{L-j}
$$

or

$$
H_{\mathrm{Lj}}=P_{\mathrm{j}} D_{\mathrm{L}-\mathrm{j}}^{(K-1)}
$$

Therefore

$$
D_{\mathrm{L}}^{(K)}=\sum_{\mathrm{j}=0}^{\mathrm{L}}\left(\begin{array}{c}
L \\
j
\end{array}\right) P_{\mathrm{j}} D_{\mathrm{L}-\mathrm{j}}^{(K-1)}
$$

We are able, therefore, to express $D_{L}^{(K)}$ as a bilinear combination of the moments of $Q^{(K-1)}$ and $P$ of order less than or equal to $L$. For example,

$$
\begin{aligned}
& D_{0}^{(K)}=P_{0} D_{0}^{(K-1)}, \\
& D_{1}^{(K)}=P_{0} D_{1}^{(K-1)}+P_{1} D_{0}^{(K-1)}, \\
& D_{2}^{(K)}=P_{0} D_{2}^{(K-1)}+2 P_{1} D_{1}^{(K-1)}+P_{2} D_{0}^{(K-1)},
\end{aligned}
$$

etc. From the definition it is clear that

$$
Q^{(1)}(N)=P(N)
$$

It is straightforward to show, by induction, that for any $K>0$ we have

$$
\begin{aligned}
& D_{0}^{(K)}=\left(P_{0}\right)^{K}, \\
& D_{1}^{(K)}=K P_{0}^{K-1} P_{1}, \text { and } \\
& D_{2}^{(K)}=K P_{0}^{K-1} P_{2}+K(K-1) P_{1}^{2} P_{0}^{K-2} .
\end{aligned}
$$

The mean time of arrival $\bar{t}$ is given by

$$
\bar{t}=D_{1}^{(\dot{K})} / D_{0}^{(K)} .
$$

The fatality rate $f$ is

$$
t=D_{0}^{(K)} \text {. }
$$


The standard deviation of the arrival time about the mean is

$$
\sigma=\left[\frac{D_{2}^{(K)}}{D_{0}^{(K)}}-\left(\frac{D_{1}^{(K)}}{D_{0}^{(K)}}\right)^{2}\right]^{1 / 2}
$$

The lateral offset $L_{0}$ is

$$
L_{0}=2 \sqrt{\frac{D_{1}^{(K)}}{D_{0}^{(K)}}-1}
$$

From (13) - (15) we have

$$
\begin{aligned}
& \bar{t}=K\left(P_{1} / P_{0}\right) \\
& t=\left(P_{0}\right)^{K} \\
& \sigma=K^{1 / 2} \sqrt{\frac{P_{2}}{P_{0}}-\left(\frac{P_{1}}{P_{0}}\right)^{2}}, \text { and } \\
& L_{0}=(4 K)^{1 / 2} \sqrt{\frac{P_{1}}{P_{0}}-1}
\end{aligned}
$$

Our remaining task is to relate $P(N)$ to the obstacle density $p$ for a variable space. Suppose a vehicle has just achieved $y=j$. The probability that it will be able to move due north is clearly (1-p). Therefore

$$
P(1)=(1-p)
$$

The probability that it will not be able to move north but will be able to move at least one of east and west is $p-p^{3}$. Then

$$
P(2)=(1-p)\left(p-p^{3}\right)
$$

is the probability of moving north on the second move. In general,

$$
P(N)=(1-p)\left(p-p^{3}\right)^{N-1}, N>0 \text {. }
$$

We insert (20) into (2) and find,

$$
\begin{aligned}
& P_{0}=\frac{1-p}{1-p+p^{3}} \\
& P_{1}=\frac{1-p}{\left(1-p+p^{3}\right)^{2}} \\
& P_{2}=\frac{(1-p)\left(1+p-p^{3}\right)}{\left(1-p+p^{3}\right)^{3}}
\end{aligned}
$$

Equations (21) - (23) may be substituted directly into (16) - (19) to yield the expression given in the text. 


\section{DOCUMENT CONTROL DATA - R \& D}

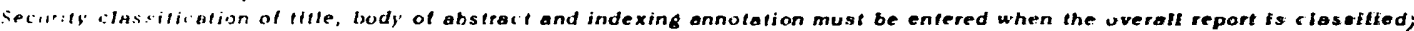

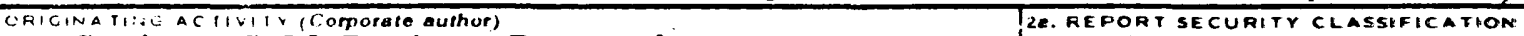
U.S. Army Cold Regions Research and Engineering Laboratory Hanover, New Hampshire 03755 HE OST TILLE

MODEI ANALYSIS OF VEHICLE TRAFFTCABILITY WITH APPLICATION

TO SURFACE EFFECT VEHICLES ON SEA ICE FIELDS

4 DESCRIPTIVE NOTES (TYPo of roport and Inclusive dates)

S AUTHOR(S) (First name, middio initlal, last namo)

Martin Smith and Yoshisuke Nakano

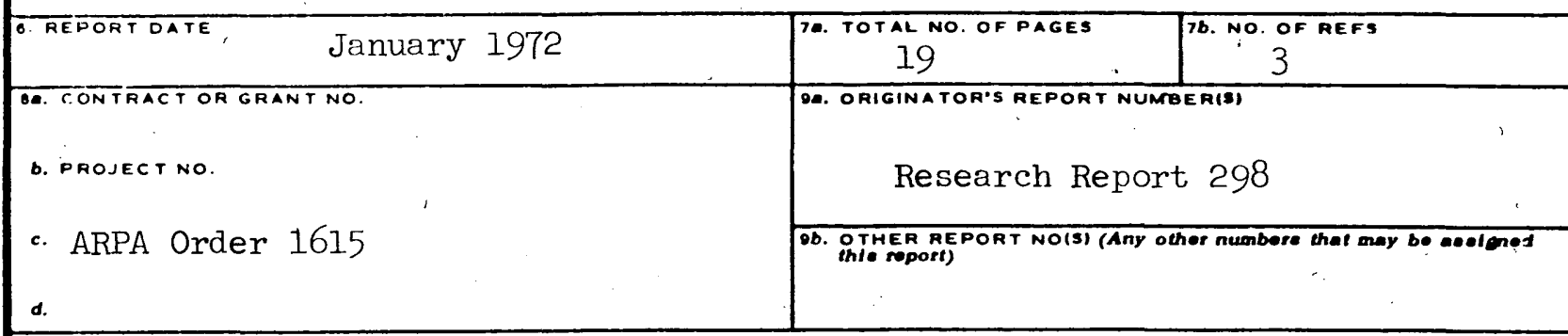

10. DISTRIBUTION STATEMENT

Approved for public release; distribution unlimited.

11. SUPPLEMENTARY NOTES 12. SPONSOAING MILITARr ÁcTivitr
Advanced Research Projects Agency

13. ADSTRACT

Efforts were made to derive the design criteria of surface effect vehicles operated on arctic sea ice. Statistical theories were developed to describe trafficability of the vehicles and topography of the sea ice. By the use of actual sea ice surface profiles obtained by an aerial laser profiler, the usefulness of the present statistical method was demonstrated.

14. Key Words

Air cushion vehicles Ground effect machines Hovercraft Ice navigation
Mathematical models

Sea ice Trafficability

Transportation models 